\section{Intravitreal injection of bevacizumab for myopic choroidal neovascularization: 1-year follow-up}

Introduction

High myopia is the most common cause of choroidal neovascularization (CNV) in adults $<50$ years of age. ${ }^{1}$ In the natural course of myopic CNV, the visual prognosis is very poor. ${ }^{2,3}$ Photodynamic therapy with verteporfin and subthreshold transpupillary thermotherapy is known to reduce the risk of severe visual acuity loss. ${ }^{4,5}$ However, neither is able to result in significant visual improvement.

Intravitreal bevacizumab seems to be an effective treatment for myopic CNV. ${ }^{6,7}$ The optimal dose range and time intervals of re-injection for long-term efficacy are largely unknown. The purpose of this study is to investigate the efficacy and visual outcome of intravitreal bevacizumab $(2.5 \mathrm{mg})$ for the treatment of myopic CNV in 12-month follow-up. new-onset CNV adjacent to the earlier lesion and visual loss of one line at the 16th month were observed in one eye. The mean VA had significantly improved from the baseline value $20 / 82$ to $20 / 25(P=0.017)$. The final mean central retinal thickness was $214.1 \pm 36.3 \mu \mathrm{m}$, with a mean decrease of $59.8 \mu \mathrm{m}$ from the pretreatment value $(P=0.017)$. The mean number of injections was 1.4 (ranging from 1 to 2) within 12 months. No other ocular or systemic side effects were noted.

Conclusions In this small series of patients with 1-year follow-up, intravitreal injection of $2.5 \mathrm{mg}$ bevacizumab seems to be effective and safe in patients with myopic CNV.

Eye (2009) 23, 2042-2045; doi:10.1038/eye.2008.404; published online 16 January 2009 Accepted in revised form: 1 December 2008 Published online: 16 January 2009

Competing interest: none.
Keywords: avastin; bevacizumab; choroidal neovascularization; intravitreal injection; myopia

\section{Materials and methods}

Chinese patients who presented with newly onset myopic CNV were included. Patients with systemic disease involving retina were excluded. This study was approved by the institutional review board. Informed consent was obtained from all patients. After full asepsis in the surgery room, anterior chamber paracentesis was carried out to lower the intraocular pressure. Then $2.5 \mathrm{mg} / 0.1 \mathrm{ml}$ bevacizumab (Avastin ${ }^{\circledR}$, Genentech, South San Francisco, CA, USA) was injected into the vitreous cavity. Pre- and post-injection examinations included assessment of BCVA (converted to LogMAR), optic coherence topography (OCT), and fluorescein angiography (FAG). FAG was repeated if any deterioration of OCT findings or visual symptoms was seen. The need for re-injection 
was assessed by changes in OCT and FAG findings, and the strategy was minimized instead of frequent fixeddose regimens. Wilcoxon signed ranks test was used for statistical analysis.

\section{Results}

In the eight eyes of the eight patients, aged 26-62 years (mean $41.5 \pm 13.0$ ), the mean spherical equivalence was $-9.8 \mathrm{D}$ (dioptre) $\pm 3.6 \mathrm{D}$ and the mean axial length was $28.3 \pm 1.8 \mathrm{~mm}$. The follow-up period ranged from 13 to 17 months (mean 14.9 months).

The mean VA had significantly improved by six lines at final follow-up ( $P=0.017$, Figure 1a). At the 12-month follow-up, vision had improved in all eyes (100\%) by three or more lines. However, a new-onset CNV adjacent to the earlier lesion and visual loss of one line at the 16th month were observed in one eye $(12 \%)$. The final mean VA had significantly improved from the baseline value $20 / 82$ to $20 / 25(P=0.017)$.

The final mean central retinal thickness was $214.1 \pm 36.3 \mu \mathrm{m}$, with a mean decrease of $59.8 \mu \mathrm{m}$ from the pre-treatment value ( $P=0.017$, Figure $1 \mathrm{~b})$. Representative patients are shown in Figures 2 and 3.

The mean number of injections was 1.4 (ranging from 1 to 2) within 12 months. No other ocular or systemic side effects were noted.

\section{Discussion}

We used re-injection treatment strategy based on changes in the OCT and FAG findings instead of frequent fixed dose regimens. The mean injection rate was $1.4 ; 3$ of 8 patients $(37.5 \%)$ received two injections and 5 of 8 patients $(63.5 \%)$ received only one injection treatment within 12 months. The second injection on three patients was performed within the first 3 months. None of the patients required any further injection from 4 to 12 months. These results suggest that infrequent dosing (1-2 doses) of bevacizumab ( $2.5 \mathrm{mg}$ ) is beneficial in the regression of myopic CNV and a high frequency of injections is not necessary in myopic patients, which holds high risk of retinal tears/detachment. Recently, lower dose levels of 1 and $1.25 \mathrm{mg}$ intravitreous bevacizumab were reported and had favourable results in 1-year follow-up. ${ }^{8,9}$ However, more frequent injections were noted. Further studies for comparing the frequency of injections using different dose levels are necessary.

The reason for using the $2.5 \mathrm{mg}$ dose of bevacizumab as opposed to 1 or $1.25 \mathrm{mg} / 0.05 \mathrm{ml}$ was to allow the greater dose to achieve sufficient therapeutic concentration in a larger volume of the vitreous chamber in the highly myopic eye. There are studies showing
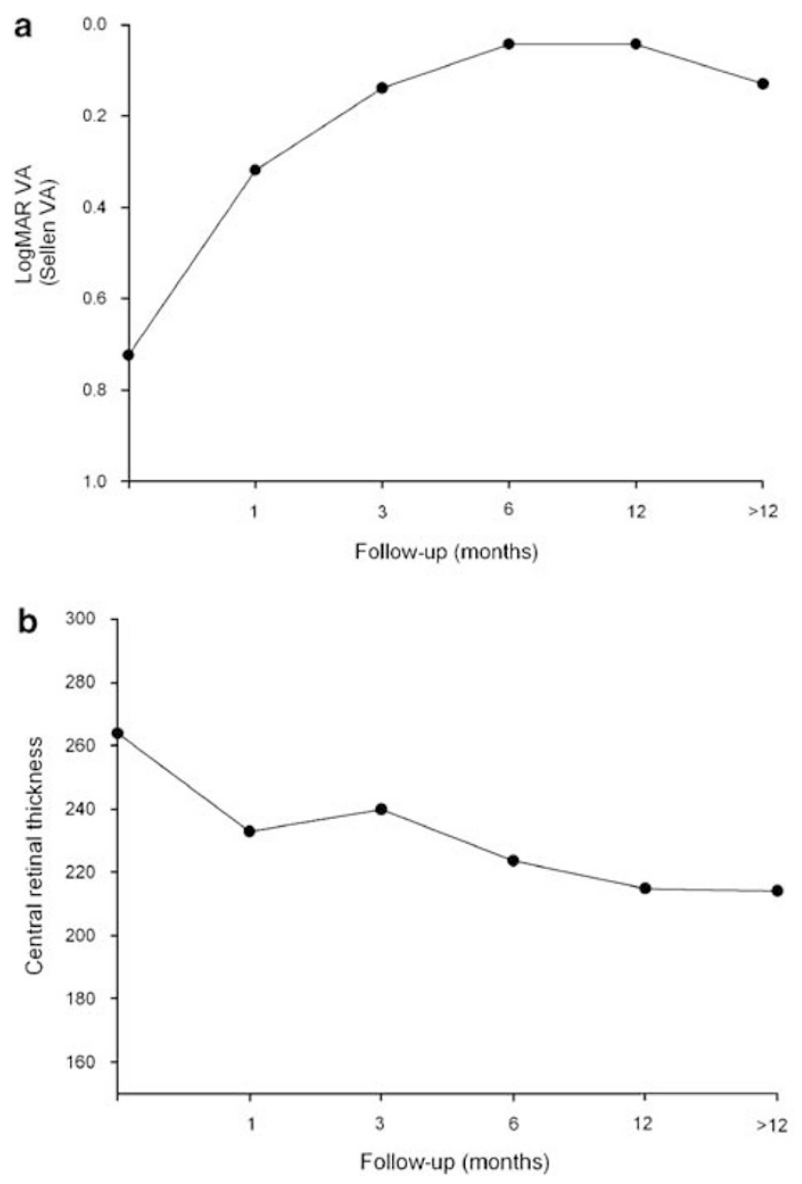

Figure 1 (a) Changes observed in the mean logMAR VA of eyes with time after treatment with intravitreal bevacizumab for myopic choroidal neovascularization $(\mathrm{CNV})$. (b) Changes observed in the central retinal thickness $(\mu \mathrm{m})$ of eyes with time after treatment with intravitreal bevacizumab for myopic CNV.

better visual acuity, in AMD treatment and less frequency of re-injection was achieved with a higher dose. ${ }^{10,11}$ We assume that the longer half-life of the larger dose of bevacizumab may help decrease the frequency of re-injection. Early treatment and younger age with relatively less choroidoretinal degeneration might also contribute to the significant vision improvement in this study.

In this study, anterior chamber paracentesis was carried out before injection to avoid drug reflux during injection and prevent elevation of intraocular pressure. Paracentesis might carry some risks of infection and lens damage. However, the incidence of these complications is very low if the procedure is carried out cautiously.

In conclusion, intravitreal bevacizumab $(2.5 \mathrm{mg})$ for myopic CNV seems to be effective for vision and anatomical improvement over 12 months. 

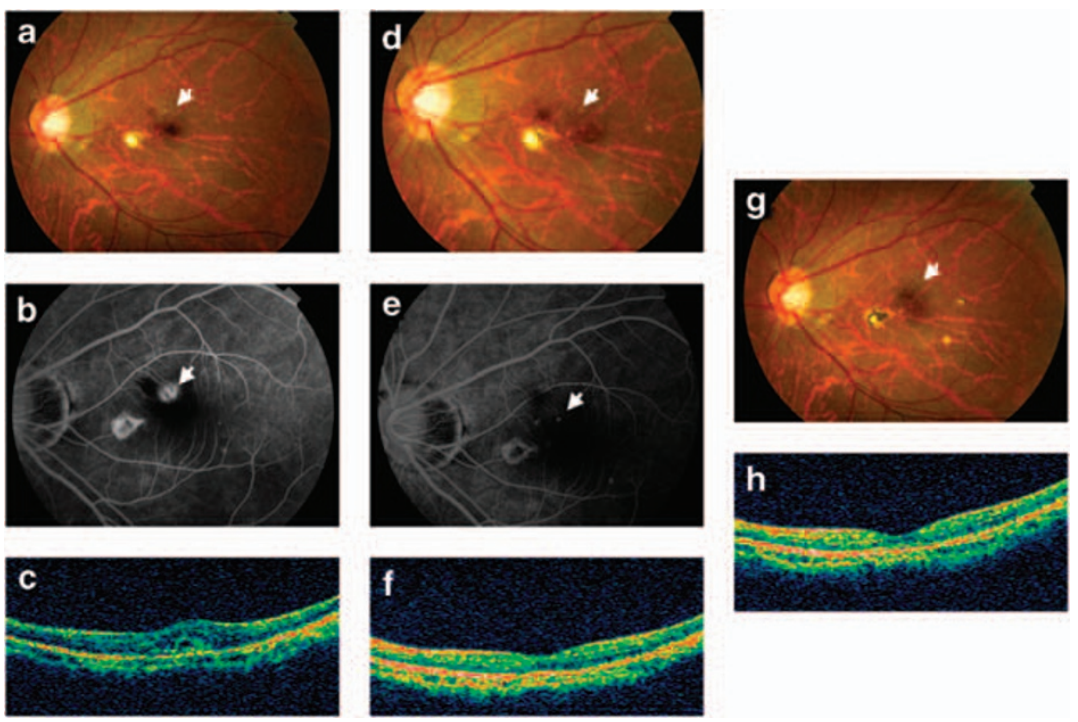

Figure 2 Left eye of a 26-year-old Chinese woman with myopia -9.75D. (a) Fundus photography revealed a juxtafoveal lesion (arrow) with subretinal haemorrhage and a nasal spot RPE atrophy area. The baseline BCVA was 20/400. (b) Late-phase fluorescein angiography (FAG) showed an ill-defined juxtafoveal choroidal neovascularization (CNV) and intense leakage of dye (arrow). (c) Optic coherence tomography (OCT) revealed an intraretinal, cystic fluid collection. (d) Two months after the first intravitreal bevacizumab treatment, this patient felt mild metamorphopsia even though vision improved to $20 / 25$. Fundus photography revealed subretinal haemorrhage resorption (arrow). (e) Late-phase FAG showed significant decrease in level of hyperfluorescence and mild hyper-fluorescence in a small CNV area (arrow). (f) OCT showed subretinal fluid resolution. Re-injection of intravitreal bevacizumab was performed in the second month. There was no complaint of metamorphopsia and no subretinal fluid in OCT during the third to seventh month follow-up and the BCVA improved to 20/20. (g, h) Fundus photography and OCT revealed no subretinal haemorrhage (arrow) or subretinal fluid in seventeenth month.
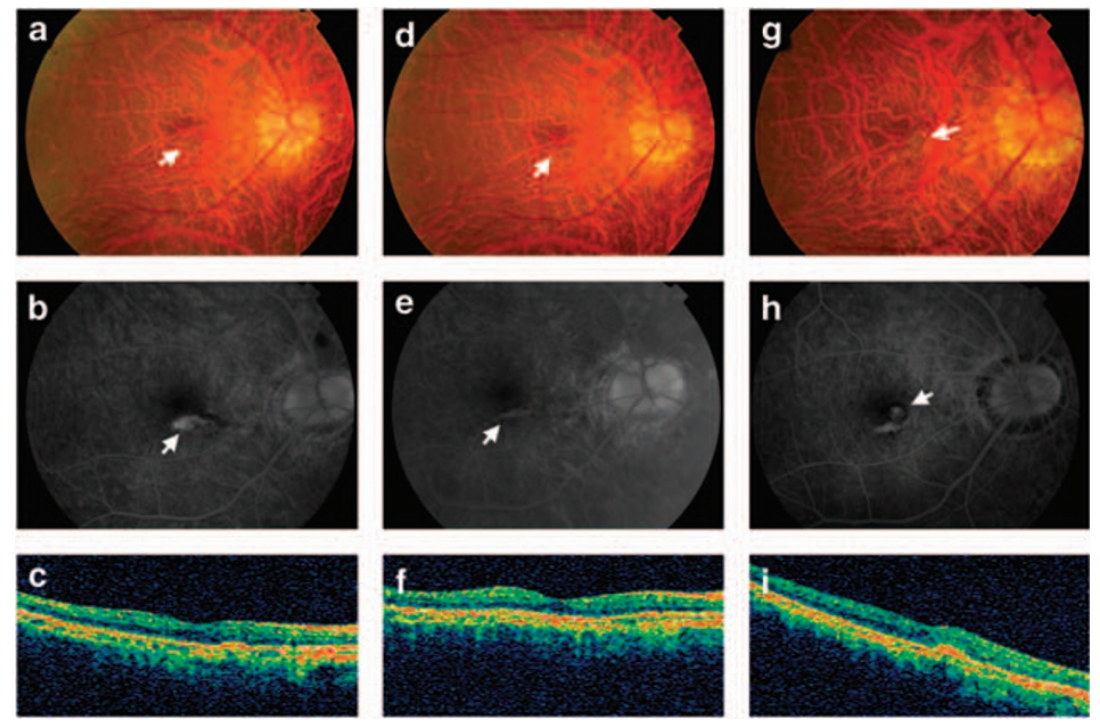

Figure 3 Right eye of a 40-year-old Chinese male with myopia -9D. (a) Fundus photography revealed a juxtafoveal lesion (arrow) with surrounding subretinal haemorrhage. The baseline BCVA was 20/66. (b) Fluorescein angiography (FAG) showed a linear patch of well-defined choroidal neovascularization (CNV) (arrow). (c) Optic coherence tomography (OCT) showed a hyper-reflective lesion under thickening retina compatible with myopic CNV. (d) Within 3 months after two injections of intravitreal bevacizumab, fundus photography revealed subretinal haemorrhage resolution (arrow) and vision improved to 20/22. (e) FAG showed mild hyperfluorescence in the CNV area (arrow). (f) OCT showed resolution of hyper-reflective lesion. There was no further visual complaint and vision improved to 20/20 during the fourth to the fifteenth month. Unfortunately, the patient complained of visual deterioration and vision decrease to 20/100 on the sixteenth month. (g) Fundus photography revealed a new grey lesion adjacent to the earlier lesion (arrow). (h) FAG showed a newly well-defined CNV lesion (arrow) adjacent to the earlier CNV area. (i) OCT showed a new hyper-reflective lesion and local retinal thickness. 


\section{References}

1 Cohen SY, Laroche A, Leguen Y, Soubrane G, Coscas GJ. Etiology of choroidal neovascularization in young patients. Ophthalmology 1996; 103(8): 1241-1244.

2 Tabandeh H, Flynn Jr HW, Scott IU, Lewis ML, Rosenfeld PJ, Rodriguez F et al. Visual acuity outcomes of patients 50 years of age and older with high myopia and untreated choroidal neovascularization. Ophthalmology 1999; 106(11): 2063-2067.

3 Yoshida T, Ohno-Matsui K, Ohtake Y, Takashima T, Futagami S, Baba T et al. Long-term visual prognosis of choroidal neovascularization in high myopia: a comparison between age groups. Ophthalmology 2002; 109(4): 712-719.

4 Blinder KJ, Blumenkranz MS, Bressler NM, Bressler SB, Donato G, Lewis $\mathrm{H}$ et al. Verteporfin therapy of subfoveal choroidal neovascularization in pathologic myopia: 2-year results of a randomized clinical trial-VIP report no. 3 . Ophthalmology 2003; 110(4): 667-673.

5 Wu P, Chen Y, Chen C, Chen YH, Kao ML, Shin SJ et al. Subthreshold transpupillary thermotherapy in Chinese patients with myopic choroidal neovascularization: 1 and 2 year follow-up. Clin Exp Ophthalmol 2008; 36(5): 443-448.

6 Chan WM, Lai TY, Liu DT, Lam DS. Intravitreal bevacizumab (Avastin) for myopic choroidal neovascularization: six-month results of a prospective pilot study. Ophthalmology 2007; 114(12): 2190-2196.

7 Hernandez-Rojas ML, Quiroz-Mercado H, Dalma-Weiszhausz J, Fromow-Guerra J, Amaya-Espinosa A, Solís-Vivanco A et al. Short-term effects of intravitreal bevacizumab for subfoveal choroidal neovascularization in pathologic myopia. Retina 2007; 27(6): 707-712.

8 Gharbiya M, Allievi F, Mazzeo L, Gabrieli CB. Intravitreal bevacizumab treatment for choroidal neovascularization in pathologic myopia: 12-month results. Am J Ophthalmol 2009; 147(1): 84-93.

9 Ikuno Y, Sayanagi K, Soga K, Sawa M, Tsujikawa M, Gomi F et al. Intravitreal bevacizumab for choroidal neovascularization attributable to pathological myopia: one-year results. Am J Ophthalmol 2009; 147(1): 94-100.

10 Costa RA, Jorge R, Calucci D, Cardillo JA, Melo Jr LA, Scott IU. Intravitreal bevacizumab for choroidal neovascularization caused by AMD (IBeNA Study): results of a phase 1 dose-escalation study. Invest Ophthalmol Vis Sci 2006; 47(10): 4569-4578.

11 Yoganathan P, Fastenberg DM, Ferrone PJ. Intravitreal Bevacizumab 2.5 versus $1.25 \mathrm{mg}$ in Exudative Age-Related Macular Degeneration: Safety and Efficacy. Program and Abstracts of the Association for Research in Vision and Ophthalmology. Fort Lauderdale: Florida, 2007 (Abstract 166). 